\title{
Analysis of Fault Tolerant Control by using Randomized Algorithms
}

\author{
Hongbin $\mathrm{Li}^{1}$, Qing Zhao ${ }^{1 *}$
}

\begin{abstract}
Active Fault Tolerant Control Systems (FTCS) can be modeled as stochastic systems with Markovian parameters. This paper addresses the modeling and analysis of such systems by using the randomized algorithms. Such algorithms are usually used to perform the robustness analysis or robust control synthesis based on a probabilistic notion. This paper extends the methods to Jump Linear Systems (JLS) and stochastic FTCS for performance verification. It discusses how to set up a model for FTCS from the imperfect FDI parameters and how to transform it into a JLS model. Then, by combining the stationary distribution of the Markov process and specific performance at each state, it presents two algorithms to estimate probability given the performance requirement or estimate performance given the probability requirement. The performance analysis can reveal some valuable results on the influence of imperfect FDI and properties of integrated design, as illustrated in the examples.
\end{abstract}

\section{INTRODUCTION}

Fault Tolerant Control Systems (FTCS) can be classified into two categories: passive and active FTCS. Active FTCS are composed of two subsystems: the Fault Detection and Isolation (FDI) scheme and the reconfigurable controller. In history, these two parts have been studied and developed independently, which leads to the separate design in active FTCS. However, existence of uncertainty in the process model and/or unknown system disturbances usually cause imperfect decision of FDI, which may corrupt the overall stability and performance of the FTCS [1], [2]. Many researchers have investigated this issue and proposed the so-called integrated FTCS framework [3], [4], [5]. One of the widely adopted framework is to model the fault and FDI behavior by using two separate Markov processes. The behavior of imperfect FDI is generally described by time delay, false alarm rate or missing detection rate. A method to estimate these parameters, especially the false alarm rate, was proposed based on a probabilistic algorithms [6]. Nevertheless there is little effort that aims to incorporate these parameters in the modeling of FTCS.

This paper tries to find a way to calculate the transition rates of Markov process based on the parameters of FDI in order to obtain a valid model for FTCS that reflects the real performance. Another motivation is to analyze the overall performance of FTCS. By generating an integrated Markov process and setting up a Jump Linear System (JLS) model for FTCS, we propose to adopt a probabilistic approach based on the randomized algorithms for the analysis of the

\footnotetext{
1 The authors are with Department of Electrical \& Computer Engineering, University of Alberta, Edmonton, Alberta, Canada, T6G 2V4.

* The corresponding author. Tel: (780)492-5792; Fax: (780)492-1811; Email: qingzhao@ece.ualberta.ca.
}

stochastic FTCS. As we know, the modeling uncertainty is a major cause of errors in FDI schemes, and the FDI performance can be captured in terms of error probabilities. In the context of FTCS, the overall system performance is affected by modeling uncertainty indirectly through the imperfect FDI. It is meaningful and desirable to carry on the performance analysis of the overall FTCS by using probability based criteria, so that the effect of FDI error probabilities can be clearly interpreted and demonstrated. To introduce the probability notion in the active FTCS, the randomized algorithms which was originally proposed in the robust control area offer a natural and appropriate approach. The performance evaluation of the FTCS gives valuable results on the influence of imperfect FDI and the properties of integrated design of FTCS.

This paper is organized as follows. In section II, it first gives the modeling of FTCS, then discusses how to describe the imperfect FDI results. In section III, two algorithms that extend the randomized algorithms to handle JLS are presented: one is about estimating the probability given the performance requirement, and the other estimating the performance given the probability requirement. By combining the two Markov processes into a single integrated Markov process, the FTCS can be converted into JLS and the algorithms can be thereby applied to carry out the performance analysis. The numerical examples are also provided for each method.

\section{Modeling OF FTCS}

\section{A. Fault and FDI process}

Fault occurrence in a system is essentially random due to the unknown and many possible causes. By assuming a memoryless property or constant failure rate, researchers have been using a continuous-time homogenous Markov process taking value from a finite state space to describe a fault process, see [7] for details.

FDI is the scheme to detect and identify the faults in the system. It can be considered to be a stochastic hypothesis test on the residual signal generated from the discrepancies between the model and the real process. In a single sample test, if the information is discarded at each sample and the noise is white, the FDI result is considered to be memoryless, i.e., the future outcomes of the FDI are independent of the past outcomes given the present outcome [3]. So Markov process can also be used to characterize the behavior of such a FDI process, which is conditioned on the fault status of the system. 
According to the above analysis, FTCS can be modeled as the following equations with Markovian parameters.

$$
\begin{aligned}
& \dot{x}(t)=[A(\zeta(t))+\Delta A(\zeta(t))] x(t)+[B(\zeta(t))+\Delta B(\zeta(t))] \\
& \cdot u(\eta(t), t)+E(\zeta(t)) w(t), \\
& y(t)=[C(\zeta(t))+\Delta C(\zeta(t))] x(t)+[D(\zeta(t))+\Delta D(\zeta(t))] \\
& \cdot u(\eta(t), t)+F(\zeta(t)) w(t) .
\end{aligned}
$$

In the model, $x(t) \in \mathbb{R}^{n}, \quad u(\eta(t), t) \in \mathbb{R}^{m}, \quad y(t) \in \mathbb{R}^{l}$ and $w(t) \in \mathbb{R}^{h}$ represent the system state, control input, output and exogenous unknown disturbance respectively. $\zeta(t)$ and $\eta(t)$ are assumed to be two separate continuous-time Markov processes to represent the system fault process and FDI process with finite state spaces $S_{1}=\left\{0,1,2, \cdots, N_{1}\right\}$ and $S_{2}=\left\{0,1,2, \cdots, N_{2}\right\} \cdot \zeta(t)$ is a homogeneous process while the transition rates of $\eta(t)$ depend on the current state of $\zeta(t)$. This non-homogeneous property of $\eta(t)$ describes the functions of FDI, whose states follow the real fault states of the process with a random time delay and error probabilities. To distinguish the system state $x(t)$ and the Markov states, the states of the Markov processes $\zeta(t)$ and $\eta(t)$ are referred to as fault and FDI modes respectively.

$A(\zeta(t)), B(\zeta(t)), C(\zeta(t)), D(\zeta(t)), E(\zeta(t))$ and $F(\zeta(t))$ are system matrices with compatible dimensions. $\Delta A(\zeta(t))$, $\Delta B(\zeta(t)), \Delta C(\zeta(t))$ and $\Delta D(\zeta(t))$ are uncertainty matrices which are assumed to have known probabilistic distributions. The system matrices and uncertainties depend on the current fault mode and the control input depends on the FDI mode. So this model represents a set of linear systems. When fault occurs and the fault process jumps to a different mode, the system matrices change correspondingly to reflect the effects of the faults on the system model; when FDI scheme detects a fault and the FDI process jumps to a different mode, the control input changes correspondingly to reflect the reconfigurable control actions based on FDI results.

For notational simplicity, for $\zeta(t)=i, \eta(t)=j, i \in$ $S_{1}, j \in S_{2}$, denote

$$
\begin{gathered}
A_{i}=A(\zeta(t)), B_{i}=B(\zeta(t)), C_{i}=C(\zeta(t)), D_{i}=D(\zeta(t)), \\
E_{i}=E(\zeta(t)), F_{i}=F(\zeta(t)), \Delta A_{i}=\Delta A(\zeta(t)), \\
\Delta B_{i}=\Delta B(\zeta(t)), \Delta C_{i}=\Delta C(\zeta(t)), \Delta D_{i}=\Delta D(\zeta(t)), \\
u_{j}(t)=u(\eta(t), t) .
\end{gathered}
$$

For $\zeta(t)=i, i \in S_{1}$, assume the uncertainty matrices satisfy the following conditions:

$$
\begin{aligned}
& \Delta A_{i}=\bar{A}_{i L} \Sigma \bar{A}_{i R}, \quad \Delta B_{i}=\bar{B}_{i L} \Sigma \bar{B}_{i R}, \\
& \Delta C_{i}=\bar{C}_{i L} \Sigma \bar{C}_{i R}, \quad \Delta D_{i}=\bar{D}_{i L} \Sigma \bar{D}_{i R},
\end{aligned}
$$

where $\bar{A}_{i L}, \bar{A}_{i R}, \bar{B}_{i L}, \bar{B}_{i R}, \bar{C}_{i L}, \bar{C}_{i R}, \bar{D}_{i L}$ and $\bar{D}_{i R}$ are uncertainty weighting matrices. $\Sigma$ with the condition of $\|\Sigma\| \leq$ 1 is assumed to be uniformly distributed matrix in a unit ball of $l_{p}$-induced norm.
The transition probability of the fault process $\zeta(t)$ from mode $i$ to $j, i, j \in S_{1}$, in the infinitesimal time interval of $\triangle t$, is given by,

$$
p_{i j}(\triangle t)=\left\{\begin{array}{l}
\alpha_{i j} \triangle t+o(\triangle t), i \neq j \\
1+\alpha_{i i} \triangle t+o(\triangle t), i=j
\end{array}\right.
$$

where $\alpha_{i j}$ is the transition rate of the homogeneous Markov process $\zeta(t)$ and $o(\triangle t)$ denotes the high order infinitesimal. When $\zeta(t)=k, k \in S_{1}$, the transition probability of the FDI process $\eta(t)$ from mode $i$ to $j, i, j \in S_{2}$, in the infinitesimal time interval of $\triangle t$, is given by,

$$
p_{i j}(\triangle t)=\left\{\begin{array}{l}
\beta_{i j}^{k} \triangle t+o(\triangle t), i \neq j, \\
1+\beta_{i i}^{k} \triangle t+o(\triangle t), i=j,
\end{array}\right.
$$

where $\beta_{i j}^{k}$ represents the transition rate from $i$ to $j$ for the Markov process $\eta(t)$ given $\zeta(t)=k$. The transition rates are given in the generator matrices of Markov processes [15], [16].

To simplify the analysis, but without loss of generality, we firstly deal with the case that the state spaces of $\zeta(t)$ and $\eta(t)$ are equal and both take values from $\{0,1\}$, where 0 denotes fault-free situation and 1 the faulty mode. This type of FTCS is referred to as the basic case of FTCS in the sequel and the method can be extended to the systems with multiple fault modes. The behavior of $\zeta(t)$ is governed by its generator matrix $G$; the behavior of $\eta(t)$ is decided by the generator matrix $H^{0}$ or $H^{1}$ depending on the current mode of $\zeta(t)$.

$F=\left[\begin{array}{ll}\alpha_{00} & \alpha_{01} \\ \alpha_{10} & \alpha_{11}\end{array}\right], H^{0}=\left[\begin{array}{cc}\beta_{00}^{0} & \beta_{01}^{0} \\ \beta_{10}^{0} & \beta_{11}^{0}\end{array}\right], H^{1}=\left[\begin{array}{ll}\beta_{00}^{1} & \beta_{01}^{1} \\ \beta_{10}^{1} & \beta_{11}^{1}\end{array}\right]$

The function of FDI is to identify the fault mode, $\zeta(t)$. However, no perfect model and noise-free environment exist in real world. As a result, there are error probabilities associated with the FDI decisions. Such imperfect FDI decisions usually compromises the entire control system performance or even result in instability. Therefore, the characteristics and effects of imperfect FDI have to be examined. In the rest of this section, we first define the characteristic parameters of the FDI and then reveal some connections between these parameters with transition rates of the Markov chain $\eta(t)$ in the integrated FTCS framework.

It should be mentioned that the following definitions are most appropriate for the two modes case, i.e., $S_{1}=S_{2}=$ 0,1 , which only contains the normal mode 0 and one failure mode 1 . Nevertheless, they can be readily modified when considering multiple-failure cases.

Definition 1 (Detection Time Delay (DTD)): A period of time taken by the FDI process to respond to and indicate the fault mode upon its occurrence, which in this case is a random variable depending on the model uncertainty, noise and detection algorithms.

To define other parameters, we make the following assumptions: 
- $\mathrm{DTD} \leq T$, i.e., the upper bound of DTD is $T$. $T$ is the maximum time delay of FDI that can be tolerated by the system, which reflects the hard deadline concept introduced in [8]. Any detection result should be given with $T$; otherwise, there occurs a missing detection.

- The probability that the FDI mode changes consecutively within the time duration $T$ is negligible. Hereby this time $T$ is counted from the moment that the actual fault occurs.

Definition 2 (False Alarm Rate (FAR)): When the system is normal or $\zeta(t)$ is in fault-free mode, the probability that FDI $\eta(t)$ jumps to faulty modes within $T$.

Definition 3 (Missing Detection Rate (MDR)): After a fault occurs, the probability that FDI does not respond and remains in the current (fault-free) mode within $T$.

Definition 4 (Recovery Rate From False Alarm (RRFFA)): The probability that when the system is in normal mode, the FDI returns to this normal mode within a time $T$ since it jumps to the fault mode.

Definition 5 (Correct Detection Rate(CDR)): The probability that the FDI mode indicates the fault mode upon its occurrence within a maximum time delay $T$, i.e., $\eta(t)$ follows $\zeta(t)$ within time $T$.

From these definitions, FAR and RRFFA are complementary, and the same can be said for MDR and CDR. Hence approximately we write that, RRFFA + FAR $=1$ and CDR $+\mathrm{MDR}=1$.

For $\zeta(t)$, the transition rates depend on the properties of the fault and we assume they are fixed and known. However, for $\eta(t)$, the transition rates should be determined by the performance of FDI, such as FAR, MDR and DTD. Although the modeling of FTCS by using Markovian parameters is well accepted [4], and methods for estimating imperfect FDI parameters are also investigated [6], there is rare effort on how to relate these FDI parameters to those of the Markov chain used to model the FDI process. Based on the above definitions and assumptions, we are ready to reveal the relationship between the transition rates of $\eta(t)$ and the FDI parameters.

1) Consider $\beta_{01}^{0}$ as the rate at which $\eta(t)$ leaves mode 0 to mode 1 when the system $\zeta(t)$ is in mode 0 . This is the false alarm scenario, then

$$
\begin{aligned}
\mathrm{FAR} & =\operatorname{Pr}\left\{\eta\left(t_{0}\right)=0, \eta(t)=1, t-t_{0} \leq T \mid \zeta(t)=0\right\} \\
& =\int_{0}^{T} \beta_{01}^{0} e^{-\beta_{01}^{0} t} d t=1-e^{-\beta_{01}^{0} T}
\end{aligned}
$$

where Pr represents probability operation. Thus, $\beta_{01}^{0}=$ $-\frac{1}{T} \ln (1-$ FAR $)$. In the above computation, we have used the fact that the waiting time between two consecutive jumps of Markov process is exponentially distributed [15], [16].

2) $\beta_{10}^{0}$ is the rate at which $\eta(t)$ leaves mode 1 to 0 when the process is in mode 0 , so FDI recovers from its false alarm.

$$
\begin{aligned}
1-\mathrm{FAR}= & \operatorname{Pr}\left\{\eta\left(t_{0}\right)=1, \eta(t)=0,\right. \\
& \left.t-t_{0} \leq T \mid \zeta(t)=0\right\} \\
= & \int_{0}^{T} \beta_{10}^{0} e^{-\beta_{10}^{0} t} d t=1-e^{-\beta_{10}^{0} T} .
\end{aligned}
$$

Thus, $\beta_{10}^{0}=-\frac{1}{T} \ln (\mathrm{FAR})$.

3) $\beta_{01}^{1}$ is the rate of $\eta(t)$ leaving mode 0 to mode 1 , which represents the correct detection.

$$
\begin{aligned}
1-\operatorname{MDR}= & \operatorname{Pr}\left\{\eta\left(t_{0}\right)=0, \eta(t)=1,\right. \\
& \left.t-t_{0} \leq T \mid \zeta(t)=1\right\} \\
= & \int_{0}^{T} \beta_{01}^{1} e^{-\beta_{01}^{1} t} d t=1-e^{-\beta_{01}^{1} T} .
\end{aligned}
$$

Thus, $\beta_{01}^{1}=-\frac{1}{T} \ln (\mathrm{MDR})$

4) $\beta_{10}^{1}$ is the rate of $\eta(t)$ leaving mode 1 to mode 0 , which represents a missed detection.

$$
\begin{aligned}
\operatorname{MDR}= & \operatorname{Pr}\left\{\eta\left(t_{0}\right)=1, \eta(t)=0\right. \\
& \left.t-t_{0} \leq T \mid \zeta(t)=1\right\} \\
= & \int_{0}^{T} \beta_{10}^{1} e^{-\beta_{10}^{1} t} d t=1-e^{-\beta_{10}^{1} T} .
\end{aligned}
$$

Thus, $\beta_{10}^{1}=-\frac{1}{T} \ln (1-\mathrm{MDR})$.

\section{B. Integrated Markov chain and Jump Linear System}

In the above modeling, two Markov processes are used to describe the failure and FDI separately. The overall behavior of the FTCS is governed by a combination of these two Markov processes, which can be described by an integrated Markov process, denoted by $\phi(t)$. For example, for the binary state space of $S_{1}$ and $S_{2}$ in the above model, the augmented state space is $\{(0,0),(0,1),(1,0),(1,1)\}$ and each of the state in this state space has concrete physical meaning in the context of FTCS:

1) $(0,0)$ : normal case, i.e., the system has no fault and the FDI also indicates it.

2) $(0,1)$ : the false alarm case. faulty.

3) $(1,0)$ : the missing detection case.

4) $(1,1)$ : the correct detection case.

The characteristics of the integrated Markov process can be described as follows [9]. Suppose that the generator matrix of $\phi(t)$ is represented by $G=\left[\gamma_{(i j)(k l)}\right]_{4 \times 4}$, then we have:

$$
\gamma_{(i j)(k l)}=\left\{\begin{array}{l}
\alpha_{i i}+\beta_{j j}^{i}, \quad i=k, j=l \\
\beta_{j l}^{i}, \quad i=k, j \neq l \\
\alpha_{i k}, \quad i \neq k, j=l \\
0, \quad i \neq k, j \neq l .
\end{array}\right.
$$

- It is assumed that the probability of fault mode $\zeta(t)$ and FDI mode $\eta(t)$ change at the same time is negligible.

- The transition rate that the combined state remains unchanged is computed according to the property of 
exponential distribution: if $T_{1}$ and $T_{2}$ are exponentially distributed with rate $\mu_{1}$ and $\mu_{2}$, then $T_{3}=$ $\operatorname{Min}\left\{T_{1}, T_{2}\right\}$ is also exponentially distributed with mean $\mu_{1}+\mu_{2}$ [16]. Therefore, we have the first equation in (3).

- If any one sub-state of $\zeta(t)$ and $\eta(t)$ changes, the transition rate equal to the corresponding transition rate of the process.

Then, the behavior of FTCS is governed by the single Markov process $\phi(t)$, and the system described in (1) and (2) becomes a Jump Linear System(JLS):

$$
\begin{aligned}
\dot{x}(t)= & {\left[A_{\phi(t)}+\Delta A_{\phi(t)}\right] x(t)+\left[B_{\phi(t)}+\Delta B_{\phi(t)}\right] } \\
& \cdot u_{\phi(t)}(t)+E_{\phi(t)} w(t) \\
y(t)= & {\left[C_{\phi(t)}+\Delta A_{\phi(t)}\right] x(t)+\left[D_{\phi(t)}+\Delta A_{\phi(t)}\right] } \\
& u_{\phi(t)}(t)+F_{\phi(t)} w(t)
\end{aligned}
$$

Therefore one can apply the existing results for JLS to the FTCS.

\section{ALGORITHMS FOR JUMP LINEAR SYSTEMS}

The modelling uncertainties are inevitable in any systems. In FTCS, they cause the imperfect FDI which may further corrupt the overall performance of FTCS [1]. Classical robustness analysis usually considers the worst-case scenario of uncertainties, meaning that the stability and desired performance characteristics should always be satisfied with the parameters within the largest uncertain range. However, in practice, if the worst case occurs quite rarely, the designed controller is often too conservative and its performance is not satisfactory [10], [12]. On the other hand, by assuming a probabilistic distribution of the parameter uncertainties, the probability that a specific performance is satisfied can be evaluated by randomized algorithms. This alternative criterion has clear meaning in practical engineering where the required performance objectives are always associated with some minimum probability levels [13]. Therefore, it is more attractive to the practical engineers. Furthermore, by allowing the performance level to be violated but with a small probability, it can actually improve the performance level for most uncertainty range and thereby avoid the conservativeness in certain sense. In the sequel, we call this description as probabilistic performance. Here in this work, we extend the randomized algorithms for performance verification to JLS including the FTCS described in (4) and (5).

\section{A. Estimation of probability given performance requirement}

Given a performance criterion $J((t))$, which is dependent on the uncertainty $\Sigma$ and Markov state $\phi(t)$, we intend to estimate the following probability:

$$
\operatorname{Pr}\{J(t) \leq \gamma\}
$$

where $\gamma$ is constant to represent specific performance level. More precisely, in a statistical sense, given precision level $\varepsilon \in(0,1)$ and confidence level $\beta \in(0,1)$, we need an estimate $\hat{p}$ of the probability such that:

$$
\operatorname{Pr}\{|\operatorname{Pr}\{J(t) \leq \gamma\}-\hat{p}| \leq \varepsilon\} \geq 1-\beta
$$

In the following, $J(t)$ is set as the $H_{\infty}$ norm of the closedloop system at $t$. Based on the randomized algorithms in [11], the following algorithm is proposed for the model in Eq. (4)-(5).

Algorithm 1 (Probability estimation)

1) Let $\varepsilon^{\prime}=\varepsilon$, compute the Chernoff bound: $M \geq$ $\frac{\ln \left(2 / \beta^{\prime}\right)}{2 \varepsilon^{\prime 2}}$.

2) Generate $M$ samples of $\Sigma$ according to its distribution. For each fixed value, $i$, of $\phi(t)$ in its state space, the system becomes a standard state space model. Apply the method introduced in [11] to obtain the estimate $\hat{p}_{i}$ of the probability that the performance satisfies the requirement in the following sense for a particular state $i$ :

$$
\operatorname{Pr}\left\{\left|\operatorname{Pr}\{J(t) \leq \gamma\}-\hat{p}_{i}\right| \leq \varepsilon^{\prime}\right\} \geq 1-\beta .
$$

3) Compute the static distribution $\pi$ of $\eta(t)$ based on the generator matrix $G$ :

$$
\pi G=0, \quad \sum_{i=1}^{N} \pi_{i}=1 .
$$

4) Compute the probability estimate that the overall jump linear system satisfies the performance requirement:

$$
\hat{p}=\sum_{i=1}^{N} \hat{p}_{i} \cdot \operatorname{Pr}\{\phi(t)=i\} .
$$

5) Compute the bound of the estimate:

$$
\begin{aligned}
& \hat{p}_{L}=\sum_{i=1}^{N}\left(p_{i}-\varepsilon^{\prime}\right) \cdot \pi_{i} \\
& \hat{p}_{U}=\sum_{i=1}^{N}\left(p_{i}+\varepsilon^{\prime}\right) \cdot \pi_{i}
\end{aligned}
$$

such that

$$
\operatorname{Pr}\left\{\hat{p}_{L} \leq \operatorname{Pr}\{J(t) \leq \gamma\} \leq \hat{p}_{U}\right\} \geq 1-\beta
$$

6) If $\hat{p}_{L} \geq \hat{p}-\varepsilon$ and $\hat{p}_{U} \leq \hat{p}+\varepsilon$, then stop; otherwise, reduce $\varepsilon^{\prime}$ by a small ratio, compute a new Chernoff bound $M$ and then go to step 2).

\section{B. Estimation of performance given probability requirement}

Since the higher value of the performance level $\gamma$ (i.e., the more relaxed performance requirement), the easier the system can satisfy this performance requirement, there exists a monotone relationship between the probability level and the performance level. Based on this monotone relationship, given the probability level $p$, a bi-section algorithm is used to search for the minimum performance level $\gamma$ such that $\operatorname{Pr}\{|\operatorname{Pr}\{J(t) \leq \gamma\}-p| \leq \varepsilon\} \geq 1-\beta$, where $\varepsilon \in(0,1)$ 
and $\beta \in(0,1)$ are the given precision and confidence requirement.

Algorithm 2 (Performance estimation)

1) Set $\varepsilon^{\prime}=\varepsilon$, tol $=\varepsilon$, compute the Chernoff bound $\bar{M}$.

2) Generate $M \geq \bar{M}$ samples according to the distribution of $\Sigma$, and evaluate the performance of the system at each sample for every discrete state.

3) Find the maximum and minimum performance level for all the samples and for all Markov states, which is set to be the upper and lower bound of performance level: $J_{\text {up }}$ and $J_{\text {lo }}$. The corresponding probability upper bound and lower bound are: $p_{\text {up }}=1$ and $p_{\text {lo }}=0$.

4) Let $J_{m}=\left(J_{\text {up }}+J_{\text {lo }}\right) / 2$, use the previous algorithm to estimate $\hat{p}$.

5) If $\hat{p}>p, p_{\text {up }}=p, \quad J_{\text {up }}=J_{m}$; else, $p_{\text {lo }}=p, \quad J_{\text {lo }}=$ $J_{m}$.

6) If $\left|p_{\text {up }}-p_{\text {lo }}\right|<$ tol, stop; else, go to step 4).

7) Compute $J_{m}=\left(J_{\text {up }}+J_{\text {lo }}\right) / 2$ and estimate $\hat{p}$ and error bound for $J_{m}$. If it satisfies the precision requirement, stops; else, decrease $\varepsilon$ and tol by a small ratio, compute new Chernoff bound and go to step 2).

The randomized algorithms given in the section III-A and III-B can be applied to FTCS for the performance analysis. Based on the relations of the FDI parameters and the transition rates of the Markov chain, we attempt to study the effects of the model uncertainty on the FDI decision and in turn on the overall performance. For this purpose, a case study is performed, the simulation results and discussions are included in the next section.

\section{Case Study And Simulation Results}

A. Example 1: estimation of Performance for a jump linear system

Consider a simple jump linear system, the nonzero matrices in its model are given as follows:

$$
\begin{gathered}
A_{1}=\left[\begin{array}{ll}
-0.759 & -0.051 \\
-0.051 & -0.634
\end{array}\right], \quad B_{1}=\left[\begin{array}{l}
-1.234 \\
-0.288
\end{array}\right], \\
C_{1}=\left[\begin{array}{ll}
-1.717 & 0.223
\end{array}\right], \quad E_{1}=0.367 \\
A_{2}=\left[\begin{array}{ll}
-1.607 & -0.564 \\
-0.564 & -1.758
\end{array}\right], \quad B_{2}=\left[\begin{array}{c}
0 \\
0.738
\end{array}\right], \\
C_{2}=\left[\begin{array}{ll}
0 & 1.622
\end{array}\right], \quad E_{2}=0.935 \\
A_{3}=\left[\begin{array}{ll}
-0.791 & -0.503 \\
-0.503 & -1.791
\end{array}\right], \quad B_{3}=\left[\begin{array}{l}
-1.256 \\
-0.347
\end{array}\right], \\
C_{3}=\left[\begin{array}{ll}
-3.765 & -4.698
\end{array}\right], \quad E_{3}=-1.021 .
\end{gathered}
$$

The generator matrix for the Markov chain is given as:

$$
G=\left[\begin{array}{ccc}
-3 & 2 & 1 \\
0.5 & -2 & 1.5 \\
1 & 4 & -5
\end{array}\right]
$$

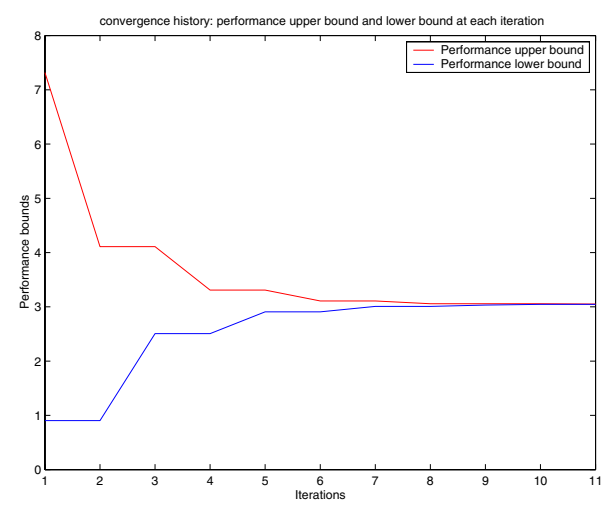

Fig. 1. Trajectory of the performance bounds.

The uncertainty weighting matrices for fault process state mode 1 are:

$$
\begin{gathered}
\bar{A}_{1 L}=\bar{A}_{1 R}=\operatorname{diag}\{0.2,0.2\} \\
\bar{B}_{1 L}=\operatorname{diag}\{0.2,0.2\}, \quad \bar{B}_{1 R}=\left[\begin{array}{ll}
0.2 & 0.2
\end{array}\right]^{T}, \\
\bar{C}_{1 L}=\left[\begin{array}{ll}
0.2 & 0.2
\end{array}\right], \quad \bar{C}_{1 R}=\operatorname{diag}\{0.2,0.2\} .
\end{gathered}
$$

where the notation, ' $\operatorname{diag}\{\cdot, \cdot\}$ ', represents a diagonal matrix with the given nonzero elements. The other uncertainty matrices for the mode 2 and 3 are of same structure but with elements set as 0.5 and 0.6 , respectively.

Following the procedures in Algorithm 1 given in Section III-A, set the $H_{\infty}$ performance level as $\gamma=3.06$, the precision $\varepsilon=0.02$ and $\beta=0.05$, we obtain the following results for the individual mode $i=1,2,3: \hat{p}_{1}=$ 0.6197, $\hat{p}_{2}=1, \quad \hat{p}_{3}=0.0035$. Then the probability estimate of the overall system for the given performance level and its bounds are: $\hat{p}=0.7172, \hat{p}_{\text {lo }}=0.6972, \hat{p}_{\text {up }}=$ 0.7285 .

This estimate can be verified by performing a simulation of the above jump linear system. The ratio between the time of satisfactory performance with the total simulation time is computed to be 0.7097 when the simulation time is 2000 seconds. It is close to the above estimate $\hat{p}$ and within the precision bounds. Hence the Algorithm 1 provides a valid estimation of the probability.

Next we intend to search for the best achievable performance level by using the bi-section algorithm when set $p=0.7, \quad \varepsilon=0.025, \quad \beta=0.05$. The results are: $\operatorname{Pr}\{|\operatorname{Pr}\{J(t)<3.0483\}-0.7|<0.025\}>0.95$. The convergence trajectories when set $\varepsilon^{\prime}=$ tol $=0.02$ are shown in the Fig. 1. These results can also be similarly verified by simulation.

\section{B. Example 2: performance evaluation of FTCS}

We now examine the closed-loop performance of FTCS for the example given in [5]. The system matrices are given as follows:

$$
A_{1}=A_{2}=\left[\begin{array}{cc}
1 & 0 \\
0 & 0.8
\end{array}\right], \quad B_{1}=\left[\begin{array}{cc}
0 & 1 \\
-0.25 & 0.25
\end{array}\right],
$$




$$
\begin{gathered}
B_{2}=\left[\begin{array}{cc}
0 & 0.2 \\
-0.25 & 0.05
\end{array}\right], C_{1}=C_{2}=\left[\begin{array}{ll}
1 & 2
\end{array}\right], \\
E_{1}=\left[\begin{array}{l}
0.05 \\
0.05
\end{array}\right], \quad E_{2}=\left[\begin{array}{c}
0.05 \\
0.1
\end{array}\right] .
\end{gathered}
$$

The uncertainty only appears in $\Delta B_{1}$ and $\Delta B_{2}$.

$$
B_{1 L}=B_{1 L}=B_{2 L}=B_{2 R}=\left[\begin{array}{cc}
0.1 & 0 \\
0 & 0.1
\end{array}\right] .
$$

The generator matrices of for failure process $\zeta(t)$ and FDI $\eta(t)$ are:

$$
F=\left[\begin{array}{cc}
-0.5 & 0.5 \\
1 & -1
\end{array}\right], \quad H^{0}=H^{1}=\left[\begin{array}{cc}
-0.1 & 0.1 \\
0.1 & -0.1
\end{array}\right] .
$$

The following two controllers in [14] are used for the FTCS.

$$
K_{1}=\left[\begin{array}{cc}
1.2018 & 8.9006 \\
-7.4239 & -2.1148
\end{array}\right], K_{2}=\left[\begin{array}{cc}
-0.22009 & 8.4035 \\
-7.7094 & -1.7643
\end{array}\right]
$$

The generator matrix $G$ of the integrated Markov process $\phi(t)$ and its static distribution $\pi$ are:

$G=\left[\begin{array}{cccc}-0.6 & 0.1 & 0.5 & 0 \\ 0.1 & -0.6 & 0 & 0.5 \\ 2 & 0 & -2.1 & 0.1 \\ 0 & 2 & 0.1 & -2.1\end{array}\right], \pi=\left[\begin{array}{llll}0.4 & 0.4 & 0.1 & 0.1\end{array}\right]$.

As we can see from $\pi$, this system mainly stay in mode $(0,0)$ and $(0,1)$, i.e., the normal mode and the false alarm mode. Now, let's look at the performance of the controller at each mode. Here, $H_{\infty}$ norm is used to describe the performance.

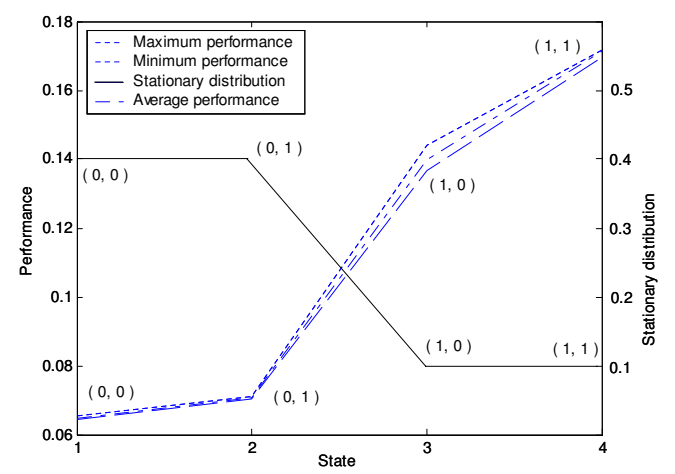

Fig. 2. Performance degradation at each mode.

The performance level for all modes are shown in Fig. 2 , together with their stationary distributions. From this figure, the performance of the system is the best in the normal mode $(0,0)$. One interesting point is that in the false alarm case, i.e., mode $(0,1)$, the system has the next-to-best performance. This is because that the system stays mainly in the modes $(0,0)$ and $(0,1)$, so the design is more tailored to cover the case when the system in normal case. On the other hand, the performance in the missing detection and correct detection cases with the controller $K_{2}$ is much worse. This is not too surprising when considering the performance degradation in the context of FTCS. When a fault occurs, even if the controller is reconfigured to compensate for such a fault, the system performance can not be completely recovered by the fault tolerant control, which reflects the performance degradation.

\section{CONCLUDING REMARKS}

In this paper, we extend the randomized algorithms to JLS and FTCS with uncertainties. The basic idea is to analyze the performance under various Markov states by the randomized algorithms and then estimate the overall performance based on the stationary distribution of Markov Chain. To analyze FTCS, we first set up the system model with two Markovian parameters considering imperfect FDI, then combine them into an integrated Markov chain and thereby transform it into a JLS model. Under such models, the above algorithms can be applied. The example shows that the integrated design can achieve a balanced performance under different states and thereby achieve better overall performance.

\section{REFERENCES}

[1] M. Mariton, "Detection delays, false alarm rates and reconfiguration of control systems", Int. J. Contr., vol. 49, pp. 981-992, 1989.

[2] M. Mahmoud, J. Jiang and Y. Zhang, "Effects of fault detection and isolation to the stability of fault tolerant control systems", Proc. American Contr. Conf., Arlington, USA, 2001.

[3] R. Srichander and B. Walker, "Stochastic stability analysis for continuous-time fault tolerant control systems," Int. J. Contr., vol. 57, no. 2, pp. 433-452, 1993.

[4] M. Mahmoud, J. Jiang and Y. M. Zhang, Active Fault Tolerant Control Systems: Stochastic Analysis and Synthesis, Springer, 2003.

[5] C. Cheng, Q.Zhao and F. Tao, "Stability and performance of the stochastic fault tolerant control systems", Proc. 42nd IEEE Conf. Decision Contr., Hawaii, USA, 2003.

[6] S. Ding, P. Zhang, P. Frank and E. Ding, "Application of probabilistic robustness technique to the fault detection system Design", Proc. 42nd IEEE Conf. Decision Contr., Hawaii, USA, 2003.

[7] A. Birolini, On the Use of Stochastic Processes in Modeling Reliability Problems, Springer-Verlag, Berlin, 1985.

[8] K. Shin and H. Kim, "Derivation and application of hard deadlines for real-time control systems", IEEE Trans. Sys., Man Cybern., vol. 22, no. 6, pp. 1403-1412, 1992.

[9] G. Kalmanovich and A. Haddad, "Optimal constant gain control of jump-linear systems with discrete state Uncertainty," Proc. 33rd Conf. Decision Contr., Lake Buena Vista, FL, December 1994

[10] R. Stengel and L. Ray, "Stochastic robustness of linear time-invariant control systems", IEEE Trans. Automat. Contr., vol.36, no.1, pp.8287, 1991.

[11] R. Tempo, E. Bai and F. Dabbene, "Probabilistic robustness analysis: explicit bounds for the minimum number of samples", Sys. Contr. Lett., vol. 30, no. 5, pp. 237-242, 1997.

[12] M. Vidyasagar, "Randomized algorithms for robust controller synthesis using statistical learning theory", Automatica, vol. 37, no. 10, pp. 1515-1528, 2001

[13] I. Yaesh, S. Boyarski and U. Shaked, "Probability-guaranteed robust $H_{\infty}$ performance analysis and state-feedback design", Sys. Contr. Lett., vol. 48, no. 5, pp. 351-364, 2003.

[14] F. Tao and Q. Zhao, "Synthesis of fault tolerant control for stochastic stability and performance," submitted to IEEE Trans. Automat. Contr.

[15] E. Çinlar, Introduction to Stochastic Processes, Prentice-Hall, Englewood Cliffs, 1975.

[16] G. Takahara, Lecture Notes on Applied Stochastic Processes, [online]available: http://www.mast.queensu.ca/ stat 455 . 